\title{
Aplicação da Análise Envoltória de Dados para avaliar a eficiência de hospitais do SUS em Mato Grosso
}

| ${ }^{1}$ Paulo Cesar de Souza, ${ }^{2}$ João Henrique G. Scatena, ${ }^{3}$ Ruth Terezinha Kehrig |

Resumo: Os hospitais são organizaçôes complexas, e um dos desafios na gestão é o aumento da eficiência. No SUS, convivem hospitais públicos e privados, emergindo a seguinte questão: qual deles é mais eficiente? Este trabalho é um estudo exploratório de natureza quantitativa, realizado em dez hospitais do SUS localizados em três regiōes de saúde do estado de Mato Grosso. A Análise Envoltória de Dados aplicada foi orientada a output, possibilitando o cálculo da Eficiência Total e Eficiência Técnica dos hospitais selecionados. O resultado mostrou que, utilizando tal modelo e nesse grupo de estabelecimentos, os hospitais privados seriam mais eficientes que os públicos, mesmo quando excluídos os mais heterogêneos. $\mathrm{O}$ uso de avaliações de eficiência hospitalar no SUS envolve ajuizamento que é dependente: do modelo adotado, das variáveis utilizadas e do contexto das unidades analisadas, destacando-se: porte, complexidade, demanda, financiamento, qualidade, vínculo funcional, entre outras especificidades. Além do mais, os resultados deste trabalho identificam pelo menos três importantes questôes que devem ser consideradas com cuidado na aplicação da Análise Envoltória de Dados a hospitais do SUS: a complexidade da avaliação da eficiência hospitalar; a escolha do método e das variáveis para tal avaliação; e como considerar o contexto em abordagens fundamentalmente quantitativas.

\author{
1 Universidade do Estado do \\ Mato Grosso. Tangará da \\ Serra-MT, Brasil (paulobbg@ \\ unemat.br). \\ ${ }^{2}$ Universidade Federal do Mato \\ Grosso, Instituto de Saúde \\ Coletiva.Cuiabá-MT, Brasil \\ (jscatena@terra.com.br). \\ ${ }^{3}$ Universidade Federal do Mato \\ Grosso, Instituto de Saúde \\ Coletiva. Cuiabá-MT, Brasil \\ (ruth.kehrig@gmail.com).
}




\section{Introdução}

Os hospitais são organizações de extrema complexidade em virtude da gama de recursos humanos, financeiros, materiais e tecnológicos que devem ser gerenciados para o alcance de seus fins. Eles operam simultaneamente em diversos níveis e com diversos objetivos, os quais são comuns aos demais componentes dos sistemas de serviços de saúde, ou seja, equidade, eficácia, eficiência, qualidade e satisfação dos usuários (MENDES, 1998).

$\mathrm{Na}$ busca por modelos gerenciais e formatos jurídicos que contemplem as peculiaridades desse tipo de organização, surgiram os diversos arranjos organizacionais existentes no Brasil. A expressão "arranjos organizacionais" é definida como "características específicas do ambiente hospitalar que influenciam o comportamento da instituição e assim, o [seu] desempenho" (LA FORGIA; CONTOLLENC, 2009, p. 180).

Os arranjos organizacionais têm implicações na propriedade do patrimônio e nas normas administrativas para o funcionamento das instâncias a que eles se referem e podem ser agrupados, segundo Braga Neto et al. (2008), em três grandes conjuntos de hospitais: o primeiro é de caráter público e estatal e integra a administração pública. O segundo é constituído no âmbito privado, mas desempenha funções de interesse público; os hospitais desse grupo integram o chamado "terceiro setor", prestando serviços à administração em conformidade com as condições estabelecidas em lei. O terceiro grupo também é de natureza privada e atua com base nas regras de mercado, podendo, mediante contrato, prestar serviços à administração pública.

Essa categorização dos arranjos organizacionais existentes no Brasil é semelhante à existente em países europeus. Analisando o sistema de saúde desses países, Saltman (2003) identificou quatro categorias com base no formato jurídico-administrativo das instituições: o público estatal, o público não estatal, o privado com fins de lucro e o privado não lucrativo.

Nas pesquisas que analisam a relação entre arranjo organizacional e eficiência nos hospitais, uma questão é sempre colocada em pauta: qual dos dois arranjos tem maior eficiência, hospitais públicos ou privados?

A eficiência (técnica) é uma medida relativa porque compara o que foi efetivamente produzido por unidade de insumo utilizado com o que poderia ter sido produzido. Assim, percebe-se que apenas a mensuração da produtividade, 
não é suficiente quando se deseja obter as informações necessárias à melhoria da eficiência, em virtude de suas limitações (FERREIRA; GOMES, 2009).

A avaliação da eficiência hospitalar justifica-se por pelo menos quatro motivos. $\mathrm{O}$ primeiro refere-se ao elevado custo da assistência hospitalar no âmbito da assistência geral à saúde. $\mathrm{O}$ segundo relaciona-se com o primeiro e versa sobre o custo de oportunidade. O terceiro é a possibilidade de os gestores utilizarem os resultados de tais avaliaçôes para analisar o impacto das políticas de saúde nos serviços hospitalares, planejar novas ações e estabelecer prioridades. O quarto motivo é que, de posse do resultado da avaliação da eficiência, os gestores hospitalares podem monitorar suas ações, bem como comparar o desempenho de determinado hospital com os demais integrantes do sistema de saúde (WOLFF, 2005).

Pergunta-se então: como calcular a eficiência de uma organização hospitalar considerando simultaneamente diversos insumos e produtos? O uso da Análise Envoltória de Dados (DEA) é uma das possibilidades.

A DEA é uma técnica não paramétrica de avaliação da eficiência relativa de um conjunto de Unidades Tomadoras de Decisão (DMU - Decision Making Units) homogêneas. Utilizando-se das quantidades de inputs consumidos e outputs produzidos por unidade e, mediante técnica de programação linear, a DEA constrói, a partir da melhor prática observada, a fronteira eficiente de produção, a qual será a base para a avaliação da eficiência das demais unidades tomadoras de decisão (LIGARDA; NACCHA, 2006). Destacou-se no meio acadêmico a partir da tese de doutorado de Edward Rhodes, publicada em 1978. O foco do trabalho era a estimativa da eficiência de escolas públicas, sem realizar o arbítrio de pesos para cada variável de entrada ou saída (GONÇALVES, 2010). Diante das múltiplas possibilidades de uso, a DEA tem sido aplicada com sucesso na análise da eficiência de organizações públicas e sem fins lucrativos, dentre as quais se destacam: escolas, faculdades, universidades, institutos de pesquisas, hospitais, clínicas, prisões, aeroportos, instituições culturais, entre outros (PENA, 2008).

Os objetivos da análise de eficiência realizada com a DEA, resumidamente, são: a) evidenciar as causas e a dimensão da ineficiência relativa de cada DMU comparada; b) gerar um indicador de eficiência; c) determinar novas metas de produção que maximizem a eficiência das DMU (JORGE et al., 2010). Existem dois fatores que influenciam significativamente os resultados obtidos quando da aplicação da DEA: modelo e orientação. 
Quanto aos modelos, existem dois que são mais amplamente utilizados: CCR e BCC. O primeiro tem sua sigla oriunda do nome dos autores Charnes, Cooper e Rhodes (1978) que publicaram o primeiro artigo sobre a DEA. O segundo é uma extensão do primeiro, como se verá adiante e tem sua sigla oriunda do nome dos autores Banker, Charnes e Cooper (1984).

O modelo CCR pressupóe retornos constantes de escala (Constant Returns to Scale-CRS) e mostra como as organizações procuram maximizar a quantidade combinada de produtos sujeitos à combinação viável de recursos utilizados. Como nem todas as organizações operam com retornos constantes de escala, o modelo CCR pode resultar em medidas de eficiência técnica distorcidas pela escala (LA FORGIA e COUTTOLENC, 2009). Diante dessa limitação do modelo CCR, surgiu o segundo modelo, chamado de BCC. Esse modelo é uma extensão do CCR e pressupõe retornos variáveis de escala (Variable Returns to Scale - VRS).

A estimativa de eficiência obtida pelo modelo CCR é chamada de "eficiência total", visto que pode ser decomposta em dois componentes: eficiência de escala e eficiência técnica interna ou eficiência pura, esta última estimada pelo modelo BCC (LA FORGIA; COUTTOLENC, 2009). No cálculo da eficiência total (CCR), compara-se uma DMU com todas as suas concorrentes. Já para o cálculo da eficiência técnica (BCC), compara-se a DMU apenas com as unidades organizacionais que operam em uma escala semelhante à sua. Neste caso, a eficiência de uma DMU é obtida dividindo-se sua produtividade pela maior produtividade dentre as DMU que apresentam o mesmo tipo de retorno à escala (ALMEIDA e MARIANO, 2006).

O segundo fator que influencia significativamente os resultados obtidos quando da utilização da DEA é a orientação aplicada ao modelo, que pode ser de duas ordens:

- Orientado a input: quando se deseja estimar qual é o mínimo nível possível de emprego de recursos, mantendo os resultados.

- Orientado a output: quando se deseja estimar qual o máximo nível possível de output mantendo fixos os input (BANDEIRA, 2000).

A seleção das variáveis (inputs e outputs) é outra etapa de grande importância, em seu aspecto qualitativo e quantitativo. Um modelo operacionalizado com muitas variáveis tende a ser benevolente, fazendo com que muitas DMU alcancem 
o score 1 de eficiência (ENCINAS, 2010). A literatura indica que o total de

Unidades Tomadoras de Decisão (DMU) deve ser no mínimo igual ao dobro do total de inputs e outputs, para garantir a aplicabilidade da análise de eficiência (FITZSIMMONS; FITZSIMMONS, 2005).

Diante da necessidade de melhoria na eficiência dos hospitais do SUS, este artigo utiliza a Análise Envoltória de Dados para avaliar, de forma comparativa, a eficiência de dez hospitais públicos e privados do SUS em Mato Grosso.

\section{Método}

Este trabalho é um estudo exploratório, com abordagem quantitativa, realizado em dez hospitais, quatro públicos eseis privados, dos quais três lucrativos e três não lucrativos. Os hospitais-caso, todos vinculados ao SUS, estão localizados em três das 16 regiōes de saúde constituídas no estado, representando 8\% dos hospitais mato-grossenses e quase $18 \%$ dos leitos SUS. Quanto ao porte, considerando os leitos totais, os hospitais são bastante variados, havendo hospitais de pequeno (três), médio (cinco) e grande porte (dois).

O número de variáveis (inputs + outputs) e de DMU utilizados na análise foi calculado segundo a orientação de Fitzsimmons e Fitzsimmons (2005), mediante a fórmula: $k \geq 2(N+M)$, onde $k$ é o número de DMU, $N$ o número de inputs e $M$ o número de outputs. Assim, segundo essa equação, o número de DMU deve ser pelo menos o dobro da soma dos inputs e outputs.

Para a aplicação da DEA, o primeiro passo realizado foi a definição de quais e quantos itens seriam considerados como inputs e outputs, visto que esta é uma das tarefas mais importantes na aplicação dessa análise. Como o total de Unidades Tomadoras de Decisão (DMU) deve ser no mínimo igual a duas vezes o total de inputs e outputs para garantir a aplicabilidade da análise de eficiência (FITZSIMMONS; FITZSIMMONS, 2005) e sendo dez o número de hospitais objetos desta pesquisa, o número de inputs e outputs utilizados respeitou o limite máximo, ou seja cinco.

O segundo passo, também de grande importância, foi a definição da orientação a ser utilizada. Optou-se pela orientação a output, a qual se justifica pelos seguintes motivos:

1 - Nos hospitais públicos, que têm participação significativa na pesquisa, os gestores têm baixa governabilidade sobre os recursos humanos, o que 
inviabiliza a utilização da orientação a input. Em geral os servidores são concursados, dificultando a redução desse input que geralmente representa por volta de $60 \%$ dos gastos para custeio e manutenção.

2-O sistema de saúde brasileiro, principalmente o público, vive uma constante busca pela garantia de mais recursos visto que a necessidade por serviços de saúde é sempre crescente diante de recursos limitados. Assim, segundo Ferreira (2009, p. 61), "não se considera factível pressupor a diminuição de recursos na área da saúde”. Para Marinho e Façanha (2001a), a orientação a output se justifica pelo fato de no caso dos hospitais, principalmente os públicos, seus principais insumos não poderem ser reduzidos facilmente.

3 - Os hospitais são organizações que possuem altos custos fixos e que muitas vezes trabalham com capacidade ociosa. Assim, esse modelo pressupõe a maximização da utilização dos recursos disponíveis, reduzindo a capacidade ociosa.

Assim, foi estabelecido o modelo empírico de hospital a ser utilizado com sua respectiva combinação de inputs e outputs. Para os inputs, foi utilizada a classificação proposta por Marinho e Façanha (2001), já consagrada em vários estudos, composta pelos seguintes itens:

- Inputs de trabalho: Número de médicos e profissionais de enfermagem (nível superior, auxiliar e técnico). A fim de melhorar essa variável, foi utilizada a ponderação do número de profissionais equivalente a tempo completo (FTE -Full Time Equivalent) (OZCAN, 1995). Assim, foi estimado quantos profissionais seriam se todos trabalhassem 40 horas semanais, ajustando as diferenças existentes de carga horária. Os dados referentes ao número de profissionais de enfermagem foram obtidos diretamente junto aos hospitais, durante a coleta de demais dados. Em relação aos médicos, os dados foram coletados do CNES, em virtude da indisponibilidade por parte dos hospitais. Foram considerados os profissionais existentes, independentemente de vínculo: contratados, concursados, terceirizados, autônomos etc.

- Inputs de capital: Número de leitos SUS. Os leitos de UTI e de isolamento são classificados como complementares pelo CNES, no entanto, também fazem parte dos inputs de capital. Neste caso, quando existentes, eles foram somados ao total de leitos gerais, visto que a utilização principalmente dos 
leitos de UTI implica altos custos para o funcionamento do hospital. A soma dos leitos de UTI aos leitos gerais foi um ajuste a fim de diferenciar os hospitais quanto ao mix de serviços, uma adaptação do que é proposto por Ozcan (1995).

- Inputs financeiros: Valor médio mensal recebido do SUS referente às internações cobradas durante o primeiro semestre de 2012. Essa variável tem sido comumente utilizada na aplicação da DEA, tendo como exemplo a pesquisa realizada por Calvo (2002) no estado de Mato Grosso, a fim de comparar a assistência hospitalar pública e privada.

Os outputs utilizados foram os seguintes:

- Internaçóes e Procedimentos de Alta Complexidade (PAC) realizados no período: Foi considerado o número médio mensal de internações SUS, somado ao número médio mensal de procedimentos de alta complexidade realizados para os pacientes internados durante o primeiro semestre de 2012, dados extraídos do SIH/SUS. Essas variáveis outputs são as mesmas utilizadas por Lobo et al. (2011), diferenciado-se no fato de que nesta pesquisa as duas variáveis foram somadas e representaram um único output, enquanto naquele elas foram utilizadas separadamente. A variável "número de procedimentos de alta complexidade" está sendo utilizada como proxy de case mix, visto que segundo La Forgia e Couttolenc (2009), esse ajuste é fundamental para a validade do modelo DEA e representa de forma aproximada a severidade ou complexidade dos casos, assim como indica utilização de um volume maior dos recursos. Essa é uma das formas possíveis de se realizar esse ajuste, sendo considerada a mais viável para esta pesquisa.

- Proxy (indicador aproximado) de qualidade: Esse indicador foi construído a partir da aplicação do instrumento de coleta de dados do Programa Nacional de Avaliação de Serviços de Saúde (PNASS) (BRASIL/MS, 2004a) e da coleta de dados estatísticos junto ao SIH/SUS. O instrumento do PNASS é composto pelas seguintes abordagens: check-list para verificação do cumprimento dos padrões de conformidade, pesquisa de satisfação com usuários e pesquisa sobre condições e relações de trabalho. Ao conjunto de indicadores oriundos do PNASS foi incorporado o inverso da Taxa de Mortalidade Geral, complementando a dimensão da qualidade, como o fez Marinho (2001). 
A ideia da utilização de um proxy de qualidade na DEA surgiu a partir do trabalho de Lobo et al. (2010). Esses autores sugerem na avaliação de hospitais universitários a inserção dessa variável relacionada à qualidade nos outputs, com inclusão de um score de satisfação dos usuários. Assim, decidiu-se pela criação de um indicador de qualidade mais completo, incorporando-se, além da satisfação do usuário, a mortalidade geral hospitalar. Neste caso, o proxy de qualidade foi composto pela média de quatro medidas: os três percentuais obtidos das abordagens do PNASS e o inverso da taxa de mortalidade geral hospitalar.

A check-list para verificação dos padrões de conformidade é composta por 22 critérios que são agrupados em três blocos: gestão organizacional, apoio técnico e logístico e gestão da atenção à saúde, blocos estes que subdividem-se em vários itens. O instrumento foi aplicado mediante visita aos diversos setores dos hospitais investigados. Os dados coletados foram organizados de modo a gerar uma nota para cada hospital, resultado da comparação entre os pontos possíveis e os pontos obtidos, em percentual.

A pesquisa de satisfação dos usuários redundou da aplicação do questionário do PNASS, na forma de entrevista, em 100 pacientes, tamanho amostral obtido pelo critério definido por esse programa (BRASIL/MS, 2004a). Os pacientes foram sorteados dentre aqueles atendidos pelo SUS que estavam internados há pelo menos um dia no hospital.

A pesquisa sobre condições e relações de trabalho foi realizada nos hospitais do estudo, abarcando 490 profissionais das categorias: médica (218), enfermagem (218) e administrativa (159). A definição da amostra respeitou o critério definido pelo PNASS e os profissionais que responderam ao questionário foram selecionados por sorteio a partir da relação de funcionários fornecida por cada estabelecimento. No entanto, os profissionais médicos tiveram a lista obtida junto ao CNES e o questionário foi aplicado àqueles que se encontravam no hospital no período de realização da pesquisa e se dispuseram a participar.

Os dados para o cálculo do inverso da taxa de mortalidade hospitalar foram extraídos do SIH/SUS, no período em análise. Pela abrangência desse sistema de informações, a mortalidade geral hospitalar calculada nos hospitais privados e filantrópicos restringiu-se ao universo de pacientes cobertos pelo SUS.

O conjunto de dados foi tabulado de forma a gerar uma nota para cada hospital, que foi transformada em percentual, podendo assim variar de 0 a $100 \%$. Para realizar a tabulação e processamento dos dados, foi utilizado o software livre 
Epidata 3.1 e o Microsoft Excel. O software Sistema Integrado de Apoio à Decisão (SIAD) foi utilizado para a aplicação da DEA.

Os dados obtidos foram organizados de modo a possibilitar a comparação dos hospitais tendo em conta a natureza jurídica, ou seja, públicos e privados (lucrativos e não lucrativos), a fim de verificar se existe diferença entre os tipos de prestador quanto à eficiência.

Esta pesquisa foi submetida ao Comitê de Ética em Pesquisa, recebendo parecer favorável sob número 45.667 de 27/06/2012 e os procedimentos realizados obedeceram aos termos estabelecidos no documento de autorização da pesquisa emitido pela direção de cada hospital. Dentre esses termos, está a não divulgação do nome do hospital, que aqui são apresentados numericamente, de forma aleatória, identificando apenas sua natureza jurídica: públicos e privados.

\section{Resultados e discussão}

A tabela 1 apresenta os valores de inputs e outputs nos dez hospitais investigados. O que primeiramente chama a atenção é a diversidade do conjunto de estabelecimentos estudados, tanto em inputs como em outputs, independentemente de sua natureza.

Tabela 1. Distribuição dos inputs e outputs entre hospitais. Mato Grosso, 2012

\begin{tabular}{l|c|ccc|cc}
\hline \multirow{3}{*}{ Prestador } & \multirow{2}{*}{ Hospitais } & \multicolumn{3}{|c|}{ Inputs } & \multicolumn{2}{c}{ Outputs } \\
\cline { 3 - 7 } & & Trabalho & Capital & Financeiro & Int.+ PAC* & Proxy \\
\hline \multirow{5}{*}{ Público } & Hospital 2 & 592 & 280 & $770.594,20$ & 725 & 38,6 \\
& Hospital 3 & 199 & 73 & $134.394,59$ & 177 & 73,2 \\
& Hospital 6 & 309 & 101 & $355.386,92$ & 471 & 68,3 \\
& Hospital 9 & 90 & 86 & $81.956,72$ & 196 & 57,3 \\
\hline \multirow{7}{*}{ Privado } & Hospital 1 & 368 & 172 & $1.232 .300,36$ & 572 & 62,6 \\
& Hospital 4 & 234 & 85 & $304.443,08$ & 374 & 67,0 \\
& Hospital 5 & 28 & 43 & $23.426,95$ & 43 & 70,5 \\
& Hospital 7 & 298 & 56 & $70.346,30$ & 132 & 70,7 \\
& Hospital 8 & 41 & 31 & $53.549,07$ & 118 & 64,9 \\
& Hospital 10 & 44 & 63 & $16.865,79$ & 29 & 60,1 \\
\hline
\end{tabular}

* PAC - Procedimentos de Alta Complexidade

Fonte: elaboração própria 
Ao se proceder à correlação de Pearson entre inputs e outputs, procedimento geralmente adotado antes da análise de eficiência (FERREIRA, 2009), observou-se correlação positiva forte entreos três inputs e o output "Internações + PAC" (tabela 2). Este é um achado que já se esperava, tendo em vista que o volume de internações (e também dos procedimentos de alta complexidade) estão relacionados ao porte do hospital, aqui expressos em número de funcionários, de leitos e o volume financeiro de seu faturamento. No caso dos hospitais estudados, utilizando-se o coeficiente de determinação, pode-se dizer que Trabalho, Capital e Faturamento determinam, isoladamente, cerca de $80 \%$ do volume de "Internaçôes + PAC".

Tabela 2. Matriz de correlação de Pearson entre inputs e outputs. Mato Grosso, 2012

\begin{tabular}{l|ccc}
\hline \multirow{2}{*}{ Outputs } & \multicolumn{3}{c}{ Inputs -2012} \\
& Trabalho & Capital & Financeiro \\
\hline Internações + PAC* $^{*}$ & 0,90 & 0,90 & 0,86 \\
Proxy de qualidade & $-0,54$ & $-0,81$ & $-0,43$ \\
\hline
\end{tabular}

* PAC - Procedimentos de Alta Complexidade

Fonte: elaboração própria

Diferentemente da situação anterior, a correlação entre os três inputs e o segundo output (Proxy de qualidade) foi negativa, embora possa ser considerada forte apenas em relação a "Capital”. A relação inversa encontrada entre porte dos hospitais e o Proxy de qualidade não encontra respaldo na literatura (BANTA; BOS, 1991; NORONHA, 2003), mas decidiu-se manter esse output no modelo porque tal correlação existe e expressa a realidade dos hospitais selecionados, o que não deve ser omitido.

É interessante refletir sobre quais as possíveis razões para essa relação inversa, contrariando a literatura que trata do assunto. A análise dos dados utilizados para o cálculo da correlação de Pearson mostra que os três hospitais com as piores médias do proxy de qualidade somam 538 leitos, ou seja, 54,34\% do total de leitos (990). Dentre esse três hospitais, dois são públicos e somam 366 leitos. Assim, percebe-se que os maiores hospitais foram os que apresentaram as menores médias do proxy de qualidade, contribuindo para este efeito contrário.

A grande questão é: quais os fatores contribuíram para que os maiores hospitais apresentassem baixo desempenho quanto à qualidade. 
Um dos fatores de grande impacto é o subfinanciamento da assistência hospitalar do estado de Mato Grosso no período estudado. Esse subfinanciamento atingiu não somente os dois hospitais públicos que estão entre os três últimos, mas também o filantrópico que tinha uma grande dependência recursos do SUS, mediante contratualização. Assim, os hospitais em análise operavam num cenário político que privilegiou a transferência de recursos para que as Organizações Sociais de Saúde gerenciassem os hospitais e deixou de transferir recursos aos municípios e mesmo aos hospitais filantrópicos para custeio e investimento (SOUZA, 2014). Tal cenário pode ser mais uma possibilidade de explicação para os resultados aqui encontrados.

Os resultados da análise de eficiência pelos dois modelos de análise do DEA (CCR e BCC), para o primeiro semestre de 2012, são apresentados na tabela 3.

Tabela 3. Resultado do cálculo de eficiência dos hospitais selecionados. Mato Grosso, 2012

\begin{tabular}{|c|c|c|c|}
\hline \multirow{2}{*}{ Hospital } & \multirow{2}{*}{ Prestador } & \multicolumn{2}{|c|}{2012} \\
\hline & & Eficiência Total (CCR) & Eficiência Técnica (BCC) \\
\hline Hospital 10 & Privado & 1,00 & 1,00 \\
\hline Hospital 9 & Público & 1,00 & 1,00 \\
\hline Hospital 8 & Privado & 1,00 & 1,00 \\
\hline Hospital 6 & Público & 1,00 & 1,00 \\
\hline Hospital 5 & Privado & 1,00 & 1,00 \\
\hline Hospital 4 & Privado & 0,98 & 1,00 \\
\hline Hospital 7 & Privado & 0,84 & 1,00 \\
\hline Hospital 1 & Privado & 0,79 & 1,00 \\
\hline Hospital 3 & Público & 0,63 & 1,00 \\
\hline Hospital 2 & Público & 0,62 & 1,00 \\
\hline
\end{tabular}

Fonte: elaboração própria

Os dados da tabela 3 evidenciam que no ano de 2012, cinco dentre os dez hospitais estudados apresentaram combinação ótima de recursos, podendo-se dizer que eles conformam a fronteira de eficiência deste universo. O hospital 4, que alcançou 0,98 pontos, também poderia ser considerado eficiente (FERREIRA, 
2009). Considerando o hospital 4 como eficiente, a tabela 4 mostra os Targets (valores alvo), ou seja, quais das variáveis de cada hospital consideradas na análise se encontram com valores abaixo ou mesmo acima do ideal.

Tabela 4. Targets (valores alvo) para os hospitais considerados ineficientes. Mato Grosso, 2012

\begin{tabular}{l|ccc}
\hline Hospital/variável & Atual & Diferença & Alvo \\
\hline Hospital 1 & & 0 & 368 \\
\hline Trabalho & 368 & 0 & 172 \\
Capital & 172 & $790.266,60$ & $442.033,76$ \\
Financeiro & $1.232 .300,36$ & 0 & 724,0 \\
Int+PAC & 572 & 166,18513 & 245,4 \\
Proxy & 62,6 & & \\
\hline Hospital 2 & & 0 & 592 \\
\hline Trabalho & 592 & 0 & 280 \\
Capital & 280 & $58.295,84$ & $712.298,36$ \\
Financeiro & $770.594,20$ & 0 & 1.175 \\
Int+PAC & 725 & 342,7 & 405,3 \\
Proxy & 38,6 & & 105 \\
\hline Hospital 3 & & 94 & 73 \\
\hline Trabalho & 199 & 0 & $134.394,59$ \\
Capital & 73 & 0 & 282 \\
Financeiro & $134.394,59$ & 0 & 146,3 \\
Int+PAC & 177 & 29,7 & $56.346,30$ \\
Proxy & 73,2 & 0 & \\
\hline Hospital 4 & & 0 & \\
\hline Trabalho & 298 & 0 & \\
Capital & 56 & $0.36,30$ & \\
Financeiro & 132 & 0,7 & \\
Int+PAC & & 0 & \\
Proxy & & 0 & \\
\hline & & 0 & \\
\hline
\end{tabular}

Fonte: elaboração própria 
Assim, para cada hospital é possível visualizar o valor encontrado, o alvo e a diferença. Não é possível comentar todos neste texto, mas destaca-se o hospital 1, como exemplo, em que o input financeiro foi uma das variáveis que contribuiu para a sua ineficiência. Os dados mostram que nesta análise, o hospital consumiu de input financeiro cerca de $64 \%$ acima do que seria considerado ideal, na combinação dos diversos inputs e outputs.

Quanto ao tipo de prestador, a composição dos hospitais considerados eficientes pelo CCR foi mista: quatro privados $(10,8,5,4)$ e dois públicos $(9,6)$. Em relação ao porte, tendo em conta os leitos SUS e a classificação do Ministério da Saúde (BRASIL/MS, 2004b), são dois hospitais de pequeno porte (10 e 5) e quatro de médio porte $(9,8,6$ e 4).

Na comparação por tipo de prestador, a média dos scores de Eficiência Total (CCR) dos hospitais privados $(0,93)$ foi maior que a média dos públicos $(0,81)$ (figura 1). Assim, utilizando este modelo de análise e aquelas variáveis inputs e outputs, entre o grupo de hospitais estudados, os privados mostraram-se mais eficientes que o públicos.

Figura 1. Média do score de Eficiência Total para os hospitais selecionados, segundo o tipo de prestador. Mato Grosso, 2012

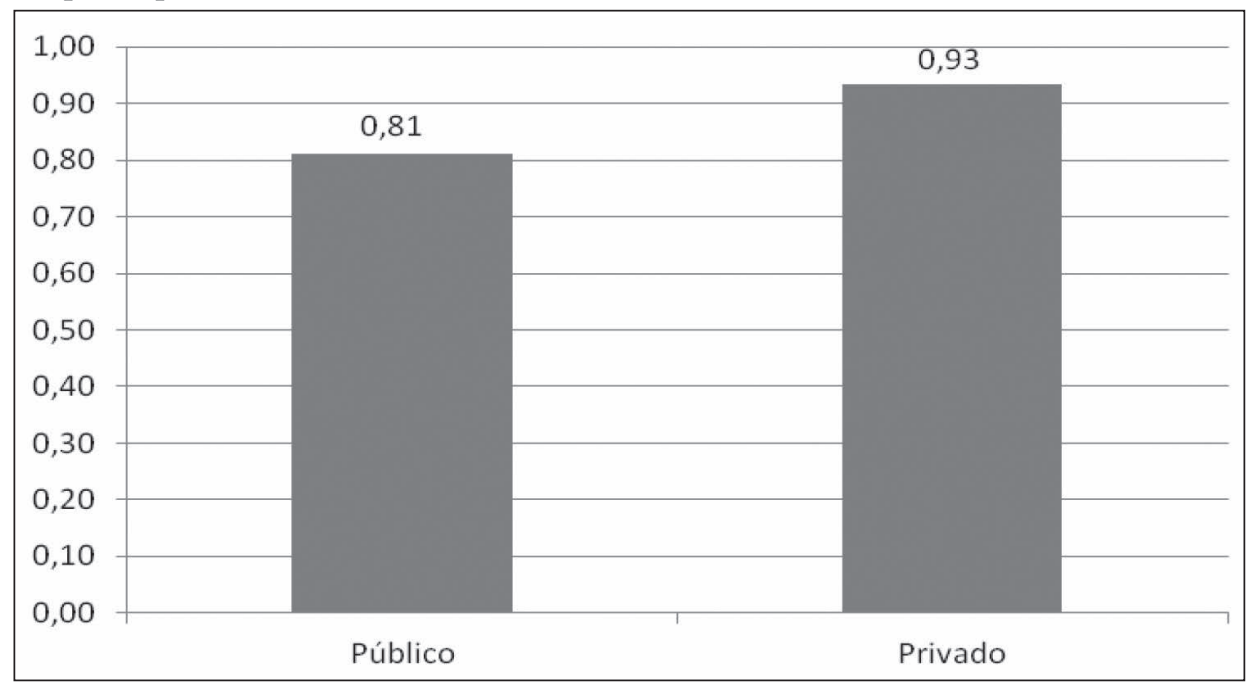

Fonte: elaboração própria

Tais resultados precisam ser analisados com cautela, pois como ressaltam Marinho e Façanha (2001), o fato de um hospital receber score de Eficiência 
Total igual a 1,00 não o converte numa ilha de eficiência, já que o grau de eficiência é apenas um indicador de consistência no balanceamento dos inputs e outputs. Além disso, eficiência máxima não significa ausência de problemas e os problemas identificados nas unidades ineficientes servem de alerta, recomendando investigações e cuidados com todo o sistema.

E como interpretar o resultado do modelo Eficiência Técnica (BCC), mediante o qual praticamente todos os hospitais investigados são eficientes?

A grande questão é que o modelo BCC parte do princípio de que os hospitais possuem realidades diversas, não sendo possível esperar retorno constante de escala (FERREIRA, 2009). Distintamente do anterior, nesse modelo, os hospitais são comparados com aqueles que operam em escala semelhante, sendo a eficiência obtida pela divisão de sua produtividade pela maior produtividade dentre os hospitais que apresentam o mesmo tipo de retorno à escala (MARIANO et al., 2006).

Assim, em virtude de os hospitais selecionados não serem homogêneos, apresentando diversidade quanto ao porte, volume de atendimentos e complexidade, elementos relacionados à escala de produção, no momento da comparação com hospitais que operam em escala semelhante, o número dos hospitais em cada nível de escala ficou muito pequeno. Desse modo, na comparação de hospitais semelhantes ocorreu um fenômeno comum nesse tipo de análise: quando o número de unidade em comparação é pequeno, tende a ser menor a capacidade do modelo em distinguir as unidades eficientes das menos eficientes (COOPER et al., 2000).

A Eficiência Técnica (BCC), ao considerar as diferenças de escala em seu cálculo e comparar entre si apenas os hospitais que operam em escala semelhante, não conseguiu, neste universo estudado, diferenciar os hospitais eficientes dos menos eficientes. Isso ocorreu por causa da diversidade dos hospitais quanto ao porte (pequeno, médio e grande), complexidade (média e alta) e volume de atendimentos (a taxa de ocupação SUS variou entre 2,6 e 82,2\%). Estes elementos determinam a escala de produção e, além disso, o número de unidades selecionadas (DMU) foi o mínimo estabelecido pela literatura. Todos esses fatores influenciaram o resultado obtido pelo modelo BCC.

Além disso, é importante destacar que nesse formato de estudo utilizou-se um número pequeno de DMUs, ou seja, o mínimo necessário a aplicação da DEA, 
limitando a resolutividade da técnica. Utilizou-se a recomendação empírica de

Fitzsimmons e Fitzsimmons (2005) de que o mínimo aceitável de DMUs deve ser igual ao dobro da soma dos inputs e outputs. No entanto, existem outros autores que recomendam que o número das DMUs deve ser de três a cinco (BANKER et al., 1989) ou de quatro a cinco (GONZÁLEZ-ARAYA, 2003) vezes a soma dos inputs e outputs. Essa é uma limitação que pode ter contribuído para a baixa resolutividade da DEA, principalmente nos resultados do modelo BCC.

Segundo Marinho e Façanha (2001), as diferenças entre os modelos CCR e BCC, principalmente no que se refere ao retorno constante ou retorno variável de escala, não se traduzem em vantagem ou desvantagem de um modelo sobre o outro. A literatura sugere que a questão crucial está na escolha dos elementos que irão compor os input e outputs.

Outra limitação importante a ser destacada refere-se à comparação entre hospitais públicos e privados (lucrativos e nãolucrativos), visto que esses hospitais operam sob lógicas diferentes. Os públicos têm um papel social a cumprir, enquanto os privados atuam sob a lógica do mercado, que é muito particular no caso dos serviços de saúde.

Aprofundando a análise, verificou-se mais uma limitação nos resultados que pode ter influenciado a ordem de classificação dos hospitais de forma individual e também a pontuação obtida por tipo de prestador.

$\mathrm{Na}$ aplicação da DEA, há um requisito já consagrado na literatura considerado de grande importância para que o método tenha um bom desempenho: a homogeneidade entre as unidades que compõem o estudo em relação às variáveis do modelo (LOBO, 2010; LOBO; LINS, 2011; FERREIRA, 2009; CALVO, 2002; LIGARDA; NACCHA, 2006). Em situaçōes em que há componentes do grupo estudado que se destacam pela heterogeneidade, Lobo et al. (2010) sugerem que sejam realizadas análises separadas de subamostras mais homogêneas.

Dois dos hospitais investigados ( 1 e 2 ) diferenciam-se dos demais por pelo menos dois fatores: porte e complexidade. $\mathrm{O}$ porte pode ser visualizado pelo número de leitos (tabela 1), visto que são considerados de grande porte aqueles que possuem de 151 a 500 leitos (BRASIL/MS, 2004b) e a complexidade pelo número de Procedimentos de Alta Complexidade (PAC) realizados. Na tabela 1, os PAC estão somados às internações, mas segundo o dados do SIH/SUS, no período analisado os hospitais 1 e 2 realizaram médias mensais de 99 e 18 PAC, 
respectivamente, enquanto na maioria dos demais não houve realização de tais procedimentos, ou ela foi de no máximo 3,3 (hospital 6).

Diante dessa constatação, repetiu-se o cálculo da Eficiência Total (CCR) e Eficiência Técnica, com exclusão dos hospitais 1 e 2, tornando assim o grupo restante mais homogêneo. Não houve qualquer alteração no resultado da Eficiência Técnica (BCC), poisa média dos scoresda Eficiência Total (CCR) alterouse discretamente, elevando-se para 0,86 (hospitais públicos) e 0,97 (hospitais privados) e a diferença entre as médias que antes era de 12 pontos reduziu para 11 pontos, alteração insignificante. Os dados mostram que, mesmo com a exclusão dos dois hospitais mais heterogêneos, a média dos scores de eficiência dos hospitais privados continua maior que a média dos públicos, mantendo-se praticamente igual a diferença entre os dois tipos de prestadores em ambas as análises.

Ressalta-se que, conforme já destacado, cada estudo tem suas particularidades e os resultados e conclusões são restritos ao grupo de hospitais analisados, sem a possibilidade de extrapolar para outros hospitais e estabelecer inferências. É importante lembrar que cada pesquisa tem suas limitaçôes e sua relevância, e deve ser interpretada dentro do contexto onde foi aplicada.

\section{Conclusão}

É interessante relembrar a questão que motivou este trabalho: qual dos dois arranjos tem maior eficiência, hospitais públicos ou privados? Os dados mostraram que, quanto à Eficiência Total (CCR), os hospitais privados mostraram-se mais eficientes que os públicos; no entanto,quanto à Eficiência Técnica (BCC), todos mostraram-se eficientes. Ressalta-se que em virtude do pequeno número de hospitais, a DEA tem sua resolutividade limitada, por isso todos os hospitais se apresentaram como eficientes. Quanto à Eficiência Total (CCR), mesmo melhorando o modelo através da eliminação dos dois hospitais mais heterogêneos, o resultado obtido anteriormente se manteve.

Ainda que aquela limitação tenha sido eliminada, permaneceram duas outras limitaçôes que devem ser destacadas. A primeira refere-se ao número de hospitais utilizados no estudo. A segunda é uma crítica à comparação entre hospitais públicos e privados, visto que funcionam sob lógicas diferentes.

Este trabalho evidenciou também os requisitos que devem ser considerados cuidadosamente no momento de se realizar avaliação da eficiência da assistência 
hospitalar no âmbito do SUS utilizando a DEA. Percebe-se que a escolha do

método e das variáveis é um passo importante, pois pode influenciar o resultado. Neste estudo, por exemplo, é possível que o resultado fosse diferente caso o método aplicado não fosse a DEA, ou mesmo se, com esse método, as variáveis input e outputs fossem outras. Outro desafio importante é como considerar o contexto de operação dos hospitais em abordagens quantitativas. Em se tratando de hospitais do SUS, elementos contextuais como organização ou desorganização do sistema, contexto sócio-político, financiamento, tempo de uso e estado de conservação dos hospitais, são elementos contextuais que podem interferir no desempenho e até explicar determinados resultados encontrados.

Espera-se que este trabalho sirva de inspiração para que outras pesquisas objetivando analisar a eficiência de hospitais do SUS sejam realizadas no estado de Mato Grosso, utilizando um número mais expressivo de hospitais, de modo a colaborar de forma ainda mais marcante para a construção do conhecimento científico relacionado à avaliação da eficiência de hospitais do SUS.

\section{Referências}

ALMEIDA, M. R.; MARIANO, E. B. A nova administração da produção: uma sequência de procedimentos pela eficiência. Seminários em Administração. Universidade de São Paulo. São Paulo, 2006. Disponível em: http://www.ead.fea.usp.br/semead/9semead/ resultado_semead/an_indicearea.asp?letra=B>. Acesso em: 16fev.2012.

BANDEIRA, D. L. Análise da eficiência relativa de departamentos acadêmicos: o caso da UFRGS. Dissertação (Mestrado) - Universidade Federal do Rio Grande do Sul. Porto Alegre, 2000.

BANTA, H. D.; BOS, M. The relation between quantity and quality with coronary artery bypass surgery. HealthPolicy, v. 18, p. 1-10, 1991.

BANKER, R.D. et al. An introduction to data envelopment analysis with some models and their uses. Research in Governmental and Non-Profit Accounting, v. 5, p. 125-163, 1989.

BANKER, R. D.; CHARNES, A.; COOPER, W. W. Some models for estimating technical and scale inefficiencies in data envelopment analysis. Management Science, v. 30, p. 1.078$1.092,1984$.

BRAGA NETO, F. C.; BARBOSA, P. R.; SANTOS, I. S. Atenção hospitalar: evolução histórica e tendências. In: GIOVANELLA, L. et al.(Org.). Políticas e sistema de saúde no Brasil. Rio de Janeiro: Fiocruz, 2008. 
BRASIL. Ministério da Saúde. Glossário do Ministério da Saúde: projeto de terminologia em saúde. Brasília: MS, 2004 b.

- Secretaria de Atenção à Saúde. Departamento de Regulação, Avaliação e Controle. Caderno do Programa Nacional de Avaliação dos Serviços de Saúde - PNASS. Brasília, DF, 2004a.

CALVO, M. C. M. Hospitais públicos e privados no Sistema Único de Saúde no Brasil: o mito da eficiência privada no estado de Mato Grosso em 1998. Tese(Doutorado) Universidade Federal de Santa Catarina,Florianópolis, 2002.

CHARNES, A.; COOPER, W. W.; RHODES, E. Measuring the efficiency of decision making units. European Journal of Operational Research, v. 2, n. 6, p. 429-444, 1978.

COOPER, W. W.; SEIFORD, L. M.; TONE, K.Data Envelopment Analysis: a comprehensive text with models, applications, references and DEA: Solver Software. Boston: KluwerAcademic Publishers, 2000.

ENCINAS, R. Oportunidades de aplicação da análise envoltória de dados em auditorias operacionais do Tribunal de Contas da União. TCC (Especialização em Orçamento Público) - Controladoria Geral da União, Brasília, 2010.

FERREIRA, C. M. C. F.; GOMES, A. P. Introdução à análise envoltória de dados: teoria, modelos e aplicaçôes. Viçosa, 2009.

FERREIRA, M. P. Assistência à saúde nos Departamentos Regionais de Saúde: um exercício metodológico sobre eficiência e acesso aos serviços de saúde. Tese (Doutorado) - Faculdade de Medicina. Universidade de São Paulo,São Paulo, 2009.

FITZSIMMONS, J. A.; FITZSIMMONS, M. J. Administração de serviços: operações, estratégia e tecnologia da informação. 4. Ed. Porto Alegre: Bookman, 2005.

GONÇALVES, A. C. Definição das restrições aos pesos em Análise Envoltória de Dados (AED) por correlação canônica e regressão linear. Tese (Doutorado) - Instituto Alberto Luiz Coimbra de pós-graduação e pesquisa em engenharia, Universidade Federal do Rio de Janeiro, Rio de Janeiro, 2010.

GONZÁLEZ-ARAYA, M.C.Projeções não radiais em regiōes fortemente eficientes da fronteira DEA - Algorítimos e Aplicaçôes. Tese (Doutorado) - COPPE, Universidade Federal do Rio de Janeiro, Rio de Janeiro, 2003.

JORGE, M. J. et al.Gestão orientada para resultado: um estudo sobre os hospitais navais da Marinha do Brasil. In: SEGeT - Simpósio de Excelência em Gestão e Tecnologia, 7.,Anais... Resende, 2010. Disponível em: http://www.aedb.br/seget/artigos2008.php

LA FORGIA, G.; COUTTOLENC, B. F. Desempenho hospitalar no Brasil: em busca da excelência. São Paulo: Singular, 2009. 
LIGARDA, L.; NACCHA, M. La eficiencia de las organizaciones de salud a través del análisis envolvente de datos. Microrredes de La Dirección de Salud IV Lima Este 2003. AnFacMed Lima, v. 67, n. 2, 2006.

LOBO, M. S. C. Aplicação da análise envoltória de dados (DEA) para apoio às políticas públicas de saúde: o caso dos hospitais de ensino. Tese (Doutorado) - Instituto Alberto Luiz Coimbra de Pós-Graduação e Pesquisa em Engenharia (COPPE), Universidade Federal do Rio de Janeiro, Rio de Janeiro, 2010.

LOBO, M. S. C.; LINS, M. P. E. Avaliação da eficiência dos serviços de saúde por meio da análise envoltória de dados. Cad. Saúde Coletiva. Rio de Janeiro, v. 19, n. 1, p. 93-102, 2011.

LOBO, M. S. C. et al. Avaliação de desempenho e integração docente-assistencial nos hospitais universitários. Revista de Saúde Pública, v. 44, n. 4, p. 581-90, 2010.

. Influência de fatores ambientais na eficiência de hospitais de ensino. Epidemiol. Serv. Saúde. Brasília,v. 20, n. 1, p. 37-45, 2011.

MARIANO, E. B.; AlMEIDA, M. R.; REBELATTO, D. A. N. Princípios básicos para uma proposta de ensino sobre análise envoltória de dados. In: COBENGE, 34., Anais... Passo Fundo: Ed. Universidade de Passo Fundo, 2006.

MARINHO, A. Avaliação da eficiência técnica nos Serviços de Saúde dos Municípios do Estado do Rio de Janeiro. Brasília, IPEA, 2001 (Texto para Discussão, 842).

MARINHO, A.; FAÇANHA, L. O. Hospitais universitários: avaliação comparativa de eficiência técnica. Rio de Janeiro, IPEA, 2001 (Texto para Discussão, 805).

MENDES, E. V. A organização da saúde no nível local. São Paulo: Hucitec, 1998.

NORONHA, J. C. de. Avaliação da relação entre volume de procedimentos e a qualidade do cuidado: o caso da cirurgia coronariana. Cadernos de Saúde Pública, v. 19, p. 1.781-1.789, 2003.

OZCAN, Y. A. Efficiency of hospital service production in local markets: the balance sheet of U.S. medical armament. Socioeconomic Planning Sciences, v. 29, n. 2, p. 139-150, 1995.

PENA, C. R. Um modelo de avaliação da eficiência da administração pública através do método Análise Envoltória de Dados (DEA). Revista de AdministraçãoContemporânea, Curitiba, v. 12, n. 1, 2008.

SALTMAN, R. B. Melting public-private boundaries in European health systems. European journal of public health, v. 13, n. 1, p. 24-9, 2003.

SOUZA, P. C. Avaliação da qualidade e eficiência da assistência hospitalar em 10 hospitais do SUS no estado de Mato Grosso. Tese (Doutorado) - Universidade Federal Mato Grosso, Cuiabá, 2014.

WOLFF, L. D. G. Um modelo para avaliar o impacto do ambiente operacional na produtividade de hospitais brasileiros. Tese (Doutorado) - Programa de Pós-Graduação em Engenharia de Produção, Universidade Federal de Santa Catarina, Florianópolis, 2005. 


\section{Abstract}

\section{Data Envelopment Analysis application to evaluate the efficiency of SUS's hospitals in the state of Mato Grosso, Brazil}

Hospitals are complex organizations, and one of their challenges is the management increased efficiency. The SUS comprises public and private hospitals, and the following question emerges: which one is more efficient? This work is an exploratory quantitative study, conducted in ten hospitals in the SUS located in three health regions of Mato Grosso state, Brazil. Data envelopment analysis (DEA) was applied oriented output, enabling the calculation of the Total Efficiency and Technical Efficiency of selected hospitals. The result showed that using this model and this group of establishments, private hospitals would be more efficient than the public, even the most heterogeneous excluded. The use of hospital efficiency ratings in the SUS involves filing that is dependent on: the adopted model, the variables used and the context of the analyzed units, particularly: size, complexity, demand, financing, quality, functional link, among other characteristics. Moreover, the results of this study identified at least three important issues that must be considered carefully in applying the DEA to SUS hospitals: the complexity of the assessment of hospital efficiency; the choice of method and the variables for such evaluation; and to consider the context in fundamentally quantitative approaches.

> Key words: health evaluation; hospital administration; organizational efficiency. 